\title{
CBS-based stabilization in explicit solid dynamics
}

\author{
Jerzy Rojek ${ }^{1, *}$, , Eugenio Oñate ${ }^{2, \ddagger}$ and Robert L. Taylor ${ }^{3, \S}$ \\ ${ }^{1}$ Institute of Fundamental Technological Research, Polish Academy of Sciences, ul. Swietokrzyska 21, \\ 00-049 Warsaw, Poland \\ ${ }^{2}$ International Center for Numerical Methods in Engineering (CIMNE), Universitat Politécnica \\ de Catalunya (UPC), Gran Capitán, $s / n$, Barcelona, Spain \\ ${ }^{3}$ Department of Civil and Environmental Engineering, University of California, Berkeley, U.S.A.
}

Paper dedicated to Professor O. C. Zienkiewicz

\begin{abstract}
SUMMARY
The characteristic-based split (CBS) stabilization procedure developed originally in fluid mechanics has been adapted successfully to solid mechanics problems. The CBS algorithm has been implemented within a finite element program using an explicit time integration scheme. Volumetric locking of linear triangular and tetrahedral elements has been successfully eliminated. The performance of the numerical algorithm is illustrated with numerical results. Comparisons with an alternative stabilization technique based on the finite calculus method also are given. Copyright (C) 2006 John Wiley \& Sons, Ltd.
\end{abstract}

KEY WORDS: CBS; volumetric locking; stabilization; dynamic analysis; explicit time integration

\section{INTRODUCTION}

Many finite elements exhibit the so-called 'volumetric locking' in the analysis of incompressible or quasi-incompressible problems in fluid and solid mechanics. Situations of this type are usual in the structural analysis of rubber materials, some geomechanical problems and most bulk metal forming processes. Volumetric locking is an undesirable effect leading to incorrect numerical results [1].

Volumetric locking in solids is present in all low-order elements based on the standard displacement formulation. The use of a mixed formulation or a selective integration technique

\footnotetext{
*Correspondence to: Jerzy Rojek, Institute of Fundamental Technological Research, Polish Academy of Sciences, ul. Swietokrzyska 21, 00-049 Warsaw, Poland.

†E-mail: jrojek@ippt.gov.pl

*E-mail: onate@cimne.upc.edu

${ }^{\S}$ E-mail: rlt@ce.berkeley.edu
} 
eliminates the volumetric locking in many elements. These methods however, fail in some elements such as linear triangles and tetrahedra, due to lack of satisfaction of the BabuškaBrezzi (BB) conditions [1-3] or alternatively the mixed patch test $[1,4,5]$ not being passed. Most linear triangular and tetrahedral elements developed within a mixed formulation also suffer volumetric locking. This poses serious limitations on the possibilities of finite element simulation of processes involving large elasto-plastic deformations like metal forming processes. Even nowadays there is hardly any meshing program that can discretize complex three-dimensional geometrical shapes of formed parts avoiding tetrahedral elements. Special stabilizing techniques must be developed to eliminate volumetric locking in linear triangles and tetrahedra.

Considerable efforts have been made in recent years to develop linear triangles and tetrahedra producing correct (stable) results under incompressible situations. Brezzi and Pitkäranta [6] proposed to extend the equation for the volumetric strain rate constraint for Stokes flows by adding a Laplacian of pressure term. A similar method was derived for quasi-incompressible solids by Zienkiewicz and Taylor [1]. Other methods to overcome volumetric locking are based on mixed displacement (or velocity)-pressure formulations using the Galerkin-least-square (GLS) method [7], average nodal pressure and average nodal deformation techniques [8,9], and sub-grid scale (SGS) methods [10-12] and the approach based on the finite calculus (FIC) formulation $[13,14]$.

The characteristic-based split (CBS) stabilization method has been developed in fluid dynamies $[15,16]$. Zienkiewicz et at. 17] have extended this technique to solid mechanics
within explicit dynanic finite element formulation. This algorithm has been further developed
to consider bulk metal forming problengs 18$]$.
In this paper, application of the CBS 2 lgorithm in solid mechanics will be reviewed. Basic
continum and discretized finite element equations are given. The constitutive model describing
large elasto-plastic deformations of metals is presented. Finally a summaty of an alternative within explicit dynan to consider bulk met

In this paper, applica continuum and discreti large elasto-plastic defo escribing stabilization algorithm based on the FIC is given [13].

of an impact problem to bulk forming problems. Numerical results obtained using the CBS method are compared with the results obtained with the FIC aigorithm and with solutions using hexahedral elements based on a mixed formulation.

\section{THE CBS ALGORITHM FOR SOLIDS}

\subsection{Continuous equations}

The CBS algorithm for solids has been derived in Reference [17]. The algorithm obtained in fluid mechanics has been extended to solid mechanics by introducing an appropriate constitutive model for a solid material in split equations describing the solid material deformation.

The problem is governed by the Stokes equations. The Stokes equations do not contain convection terms, so the term 'characteristic' in the name of the algorithm CBS is not relevant. We maintain, however, the name CBS of the algorithm developed for fluid dynamics problem governed by the Navier-Stokes equations.

Large elasto-plastic deformations are considered with a small amount of compressibility allowed, as this generally is the case with large deformation behaviour of solids. 
The equations expressing the momentum conservation are written as follows:

$$
\begin{aligned}
\rho \frac{\partial v_{i}}{\partial t} & =\frac{\partial s_{i j}}{\partial x_{j}}+\frac{\partial p}{\partial x_{i}}+b_{i} \\
\frac{\partial u_{i}}{\partial t} & =v_{i}
\end{aligned}
$$

where $\rho$ is the density, $v_{i}$ the velocity in the $i$ direction, $s_{i j}$ the deviatoric stress component, $p$ the mean stress (or pressure), $b_{i}$ the $i$ component of body force and $u_{i}$ the displacement in the $i$ direction. Equations of motion (1) are completed with appropriate boundary conditions and constitutive equations for deviatoric part of stresses $s_{i j}$ and pressure $p$. The elasto-plastic constitutive model with $J 2$ plasticity is presented later on. The constitutive law for pressure is as follows:

$$
\frac{1}{K} \frac{\partial p}{\partial t}=\frac{\partial v_{i}}{\partial x_{i}}
$$
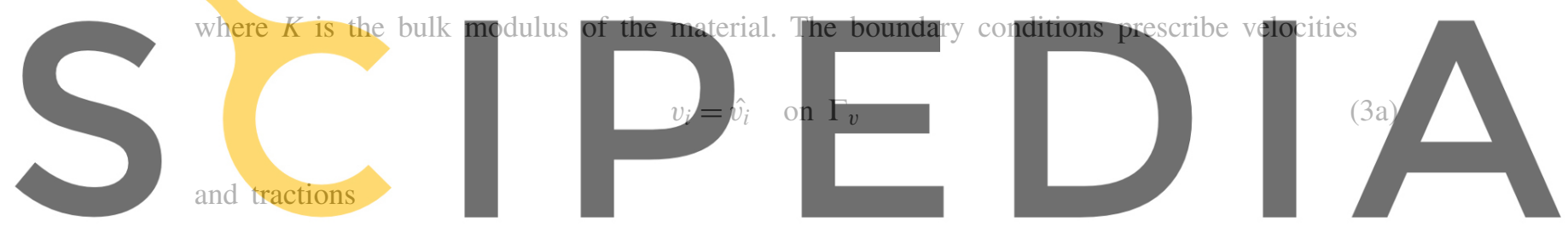

Register for free at https//www.scipedia.com to download the version without the watermark

Application of a standard, Galerkin, finite element discretization of displacement u, velocity $\mathbf{v}$ and pressure $p$

$$
\begin{aligned}
& \mathbf{u} \approx \tilde{\mathbf{u}}=\mathbf{N}_{v} \overline{\mathbf{u}} \\
& \mathbf{v} \approx \tilde{\mathbf{v}}=\mathbf{N}_{v} \overline{\mathbf{v}} \\
& p \approx \tilde{p}=\mathbf{N}_{p} \overline{\mathbf{p}}(t)
\end{aligned}
$$

to Equations (1) and (2), gives the discrete equations of mixed formulation, cf. Reference [1]

$$
\begin{aligned}
\mathbf{M} \frac{\mathrm{d}}{\mathrm{d} t} \overline{\mathbf{v}} & =\mathbf{R}_{v}-\mathbf{f}_{d}-\mathbf{C} \overline{\mathbf{p}} \\
\frac{\mathrm{d} \overline{\mathbf{u}}}{\mathrm{d} t} & =\overline{\mathbf{v}} \\
\mathbf{M}_{p} \frac{\mathrm{d} \overline{\mathbf{p}}}{\mathrm{d} t} & =\mathbf{R}_{p}+\mathbf{C}^{\mathrm{T}} \overline{\mathbf{v}}
\end{aligned}
$$


In the above, $\overline{\mathbf{v}}$ and $\overline{\mathbf{p}}$ stand for the sets of unknown nodal parameters, the matrices are given by, cf. Reference [1]

$$
\begin{aligned}
\mathbf{M} & =\int_{\Omega} \rho \mathbf{N}_{v}^{\mathrm{T}} \mathbf{N}_{v} \mathrm{~d} \Omega, \quad \mathbf{M}_{p}=\int_{\Omega} \frac{1}{K} \mathbf{N}_{p}^{\mathrm{T}} \mathbf{N}_{p} \mathrm{~d} \Omega \\
\mathbf{R}_{v} & =\int_{\Gamma_{t}} \mathbf{N}_{v}^{\mathrm{T}} \hat{\mathbf{t}} \mathrm{d} \Gamma+\int_{\Omega} \mathbf{N}_{v}^{\mathrm{T}} \mathbf{b} \mathrm{d} \Omega, \quad \mathbf{R}_{p}=\mathbf{0} \\
\mathbf{f}_{d} & =\int_{\Omega} \mathbf{B}_{v}^{\mathrm{T}} \mathbf{s} \mathrm{d} \Omega, \quad \mathbf{C}=\int_{\Omega} \mathbf{N}_{v}^{\mathrm{T}} \nabla \mathbf{N}_{p} \mathrm{~d} \Omega
\end{aligned}
$$

where $\mathbf{B}$ is the linear stress-strain operator matrix, stress deviator $\mathbf{s}$ can be determined assuming arbitrary (isotropic) constitutive model.

With a fully incompressible material $\mathbf{M}_{p}$ would be zero and this would not permit the application of time step procedures of the explicit type. Small compressibility allows us to adopt fully explicit solution scheme. In the explicit solution, the two matrices $\mathbf{M}$ and $\mathbf{M}_{p}$ are usually diagonalized.

The standard mixed formulation performs well for certain combinations of velocity and pressure discretization, which lead to finite elements satisfying BB stability conditions, like quadrilaterals and hexahedra with quadratic displacement (velocity) and linear pressure interpolations.

Elements not sati: equal-order velocity be overcome by the

While not meeting al lateral and hexahedra standard elements of nearly all commercial 'explicit' finite element programs. They will serve

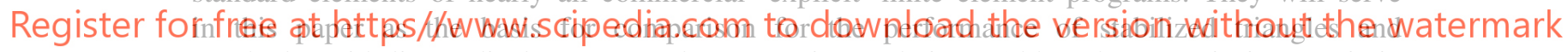
tetrahedra with linear displacement and pressure interpolations. Although our main interest is in stabilization of triangular and tetrahedral elements, the CBS algorithm will be applied to bilinear quadrilateral and trilinear hexahedral elements with equal-order displacement and pressure interpolation.

\subsection{The fractional step method}

In the fractional step method, the equations are split into parts. The sum of the parts, however, must be such that the original equations are always recovered. The split can be applied to discretized equations, cf. Reference [19]. Here we will follow a 'classical' procedure [15, 16], where the split is applied to the equations of continuum (1) and (2) following their time discretization using the generalized mid-point rule (or $\theta$ method). After temporal discretization the equations have the following form:

$$
\begin{aligned}
\rho \frac{v_{i}^{n+1}-v_{i}^{n}}{\Delta t} & =\frac{\partial s_{i j}^{n}}{\partial x_{j}}+\frac{\partial p^{n+\theta_{2}}}{\partial x_{i}}+b_{i}^{n} \\
\frac{u_{i}^{n+1}-u_{i}^{n}}{\Delta t} & =v_{i}^{n+\theta_{3}}
\end{aligned}
$$


and

$$
\frac{1}{K} \frac{p^{n+1}-p^{n}}{\Delta t}=\frac{\partial v_{i}^{n+\theta_{1}}}{\partial x_{i}}
$$

where $\Delta t=t^{n+1}-t^{n}, n$ and $n+1$ are consecutive time instants, and $\theta_{j}$ are generalized midpoint rule discretization parameters. Note that the deviatoric stress and body force terms in Equation (7a) are evaluated at different temporal points than the pressure. This is to facilitate the split introduced next. For an arbitrary variable $\phi$, we have the following relationship:

$$
\phi^{n+\theta_{j}}=\left(1-\theta_{j}\right) \phi^{n}+\theta_{j} \phi^{n+1}
$$

with $0 \leqslant \theta_{j} \leqslant 1$. In our case, $\theta_{1}$ can vary between 0.5 and 1 , while $\theta_{2}$ and $\theta_{3}$ can vary between 0 and 1 . In all that follows we shall use $\theta_{1}=1$.

The momentum equation (7a) is rewritten in the following form:

$$
\rho \frac{v_{i}^{n+1}-v_{i}^{*}+v_{i}^{*}-v_{i}^{n}}{\Delta t}=\frac{\partial p^{n+\theta_{2}}}{\partial x_{i}}+\frac{\partial s_{i j}^{n}}{\partial x_{j}}+b_{i}^{n}
$$
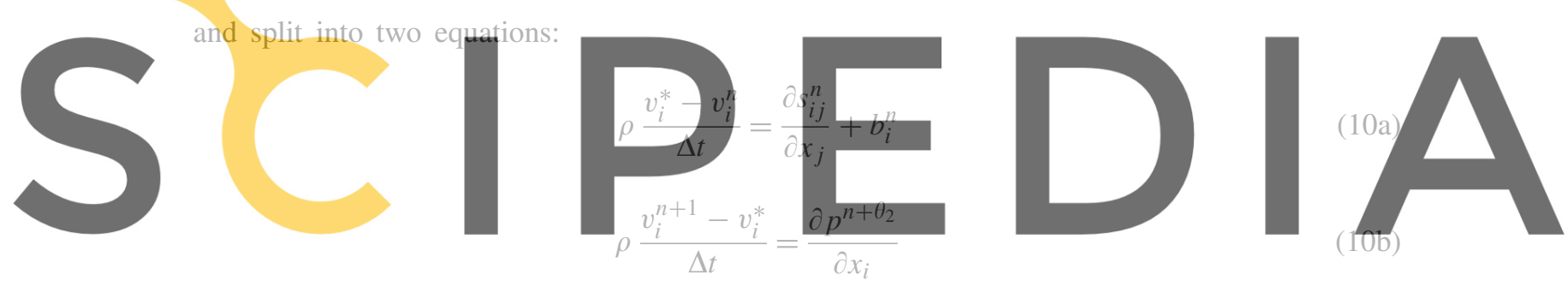

Register for free at https/fwww scipedia.GPm the download the version without the watermark obtained from Equation (10a)

$$
v_{i}^{*}=v_{i}^{n}+\frac{\Delta t}{\rho} \frac{\partial s_{i j}^{n}}{\partial x_{j}}+\Delta t b_{i}^{n}
$$

and the real velocity is calculated from Equation (10b) as

$$
v_{i}^{n+1}=v_{i}^{*}+\frac{\Delta t}{\rho} \frac{\partial p^{n+\theta_{2}}}{\partial x_{j}}
$$

Substituting Equation (11b) into Equation (7b),

$$
\frac{1}{K} \frac{p^{n+1}-p^{n}}{\Delta t}=\frac{\partial v_{i}^{*}}{\partial x_{i}}+\frac{\Delta t}{\rho} \frac{\partial^{2} p^{n+\theta_{2}}}{\partial x_{i}^{2}}
$$

Finally, from the second of Equation (7a), we obtain

$$
u_{i}^{n+1}=u_{i}^{n}+\Delta t v_{i}^{n+\theta_{3}}
$$


The CBS algorithm stated above consists of the following four steps:

(i) Calculate the fractional velocity $v_{i}^{*}$ from Equation (11a).

(ii) Calculate the pressure $p^{n+1}$ from Equation (11c).

(iii) Calculate the real velocity $v_{i}^{n+1}$ from Equation (11b).

(iv) Calculate the displacement $u_{i}^{n+1}$ from Equation (11d).

\subsection{Finite element discretization}

Introducing the finite element space discretization of pressure and velocities given by Equation (4) and applying the Galerkin method to Equations (11a), (11c) and (11b) leads to the following set of discrete equations:

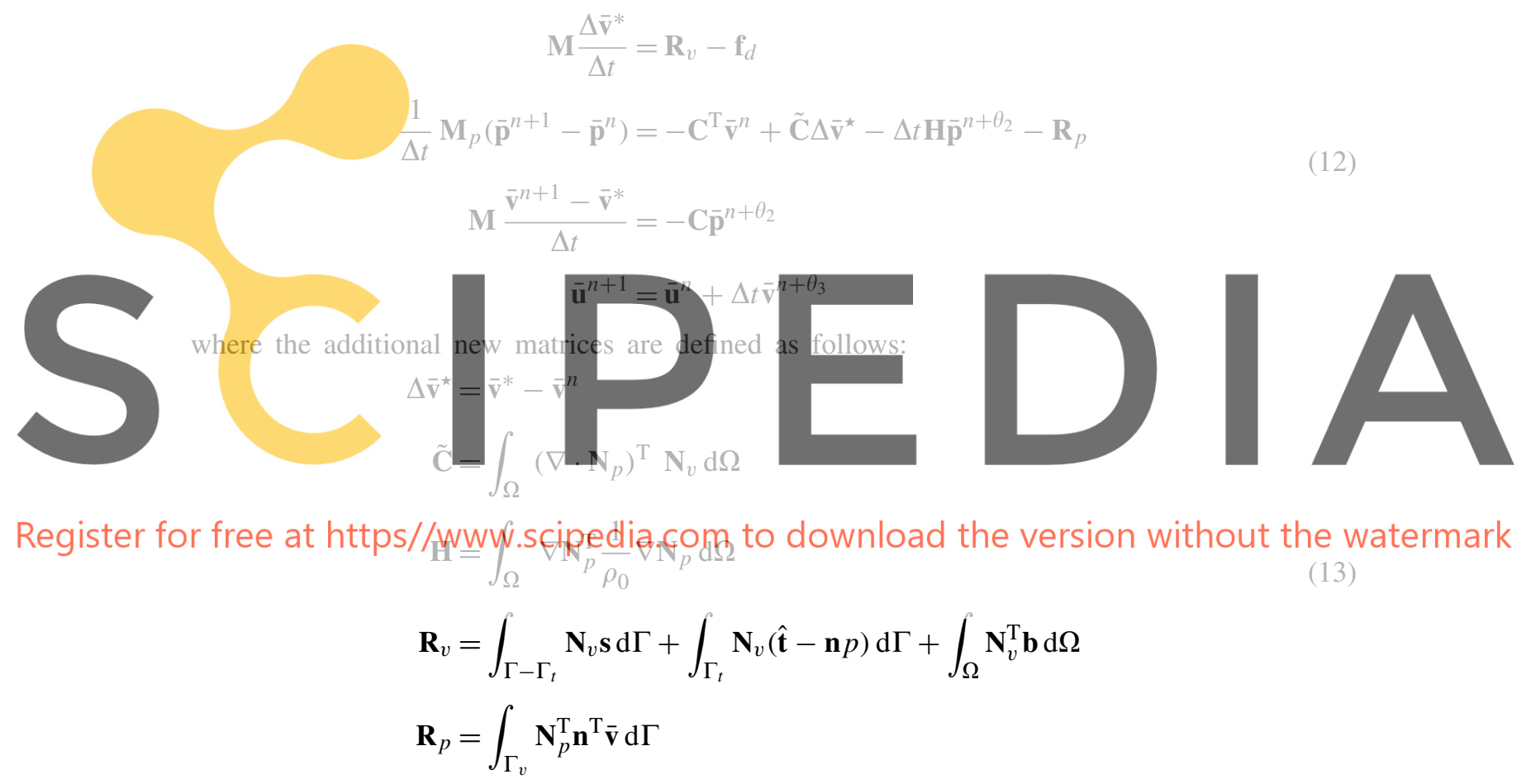

Evaluation of the second integral in $\mathbf{R}_{v}$ requires the 'extraction' of the pressure part from the prescribed traction on the part of the boundary $\Gamma_{t}$. This can be done taking the pressure from the previous time step as an estimation. A similar estimation must be made for the deviatoric stresses $\mathbf{S}$ on the part of the boundary $\Gamma-\Gamma_{t}$ to calculate the first integral in $\mathbf{R}_{v}$. In a simplified algorithm, these troublesome calculations are avoided and the vectors $\mathbf{R}_{v}$ and $\mathbf{R}_{p}$ are evaluated according to the second of Equation (6). This simplification implies the non-physical boundary condition

$$
\frac{\partial p}{\partial n}=0 \quad \text { on } \Gamma
$$

and introduces a certain error in the pressure. It can be demonstrated, however, that this error is localized to narrow boundary areas, cf. Reference [20]. This simplification is employed in the 
numerical algorithm presented below. Using this form of the algorithm, one avoids computation of $\tilde{\mathbf{C}}$, as well as, avoids integrations of the projections of deviatoric stresses on the velocity boundaries, cf. Reference [17].

\subsection{Solution algorithm}

(i) Approximate velocity increment determination:

$$
\Delta \mathbf{v}^{\star}=\Delta t \mathbf{M}^{-1}\left(\mathbf{R}_{v}^{n}-\mathbf{f}_{d}^{n}\right)
$$

(ii) The pressure increment evaluation:

$$
\left(\frac{1}{\Delta t} \mathbf{M}_{p}+\theta_{2} \underline{\Delta t} \underline{\mathbf{H}}\right) \underline{\Delta} \overline{\mathbf{p}}=-\mathbf{C}^{\mathrm{T}} \overline{\mathbf{v}}^{\star}-\underline{\Delta t} \underline{\mathbf{H}}^{n}
$$

(iii) The velocity correction:

$$
\Delta \overline{\mathbf{v}}=\Delta \overline{\mathbf{v}}^{\star}-\Delta t \mathbf{M}^{-1} \mathbf{C} \overline{\mathbf{p}}^{n+1}
$$

(iv) The displacement update:

$$
\overline{\mathbf{u}}^{n+1}=\overline{\mathbf{u}}^{n}+\Delta t \overline{\mathbf{v}}^{n+\theta_{3}}
$$

$(15 \mathrm{~d})$

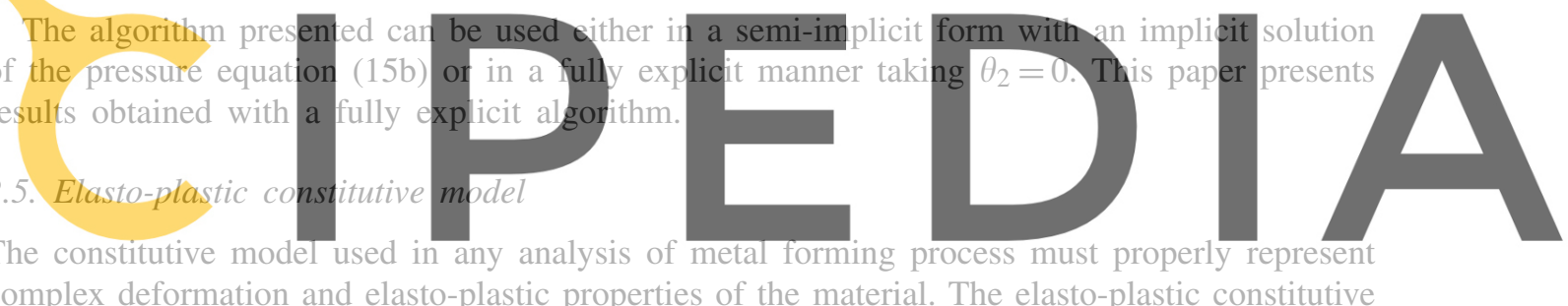

complex deformation and elasto-plastic properties of the material. The elasto-plastic constitutive

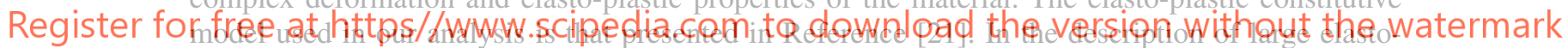

plastic deformations, the multiplicative decomposition of the deformation gradient tensor $\mathbb{F}$ into its elastic $\mathbb{F}^{\mathrm{e}}$ and plastic part $\mathbb{F}^{\mathrm{p}}$ is assumed

$$
\mathbf{F}=\mathbf{F}^{\mathrm{e}} \mathbf{F}^{\mathrm{p}}
$$

The model is developed in a stress-free intermediate configuration, and then all the constitutive relationships are transformed to the deformed configuration.

In the deformed configuration, the following additive decomposition of the Almansi strain tensor $\mathbf{e}$ into the elastic and plastic parts, $\mathbf{e}^{\mathrm{e}}$ and $\mathbf{e}^{\mathrm{p}}$, respectively, is obtained:

$$
\mathbf{e}=\mathbf{e}^{\mathrm{e}}+\mathbf{e}^{\mathrm{p}}
$$

The Almansi strain tensor, and its elastic and plastic parts, $\mathbf{e}, \mathbf{e}^{\mathrm{e}}$ and $\mathbf{e}^{\mathrm{p}}$, can be expressed by the deformation gradient tensor $\mathbf{F}$ and its elastic part $\mathbf{F}^{\mathrm{e}}$ and plastic part $\mathbf{F}^{\mathrm{p}}$ in the following form:

$$
\begin{aligned}
& \mathbf{e}^{\mathrm{e}}=\frac{1}{2}\left(\mathbf{I}-\mathbf{F}^{\mathrm{e}-\mathrm{T}} \mathbf{F}^{\mathrm{e}-1}\right) \\
& \mathbf{e}^{\mathrm{p}}=\frac{1}{2}\left(\mathbf{F}^{\mathrm{e}-\mathrm{T}} \mathbf{F}^{\mathrm{e}-1}-\mathbf{F}^{-\mathrm{T}} \mathbf{F}^{-1}\right) \\
& \mathbf{e}=\frac{1}{2}\left(\mathbf{I}-\mathbf{F}^{-\mathrm{T}} \mathbf{F}^{-1}\right)
\end{aligned}
$$


The implementation of the general model has been simplified by the assumption that elastic strains are small, which for metals is fully justified. This allows us to assume a simple form of the elastic part of the free energy function $\psi^{e}$

$$
\psi^{\mathrm{e}}\left(\mathbf{e}^{\mathrm{e}}\right)=\lambda \operatorname{tr}\left(\mathbf{e}^{\mathrm{e}}\right)^{2}+\mu\left(\mathbf{e}^{\mathrm{e}}: \mathbf{e}^{\mathrm{e}}\right)
$$

where $\lambda$ and $\mu$ are the Lamé constants. With this form of the elastic potential, the Kirchhoff stress tensor $\boldsymbol{\sigma}$ is obtained as

$$
\boldsymbol{\sigma}=\frac{\partial \psi^{e}\left(\mathbf{e}^{\mathrm{e}}\right)}{\partial \mathbf{e}^{\mathrm{e}}}=\lambda \operatorname{tr}\left(\mathbf{e}^{\mathrm{e}}\right)+2 \mu \mathbf{e}^{\mathrm{e}}
$$

In the present implementation of the model, the associated flow rule is assumed with the Huber-Mises yield criterion. The stress-strain curve is taken in the following form:

$$
\sigma_{\mathrm{Y}}=K\left(a+\overline{\mathrm{c}}^{\mathrm{p}}\right)^{n}
$$

where $\sigma_{\mathrm{Y}}$ is the yield stress and $K, a$ and $n$ are the material constants.

\section{STABILIZATION BASED ON FIC}

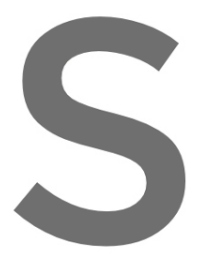

\section{The FIC approach h methods for a wide ideas were applied in and incompressible \\ The basis of the

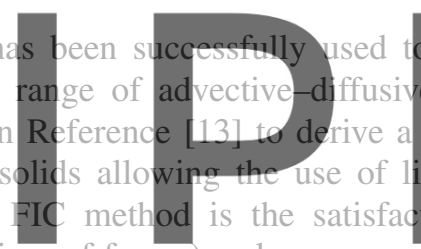 \\ momentum (equilibrium of forces) and mass conservation in a domain of finite size and retaining \\ higher order terms in the Taylor expansions used to express the different terms of the differential

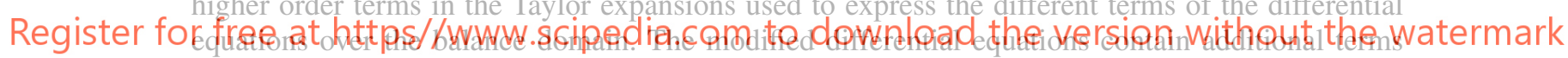 \\ which introduce the necessary stability in the equations to overcome the volumetric locking problem.}

\subsection{Equations of motion}

Within the framework of a FIC formulation, the equations of motion for a solid material are written as, cf. Reference [13]

$$
r_{i}-\frac{h_{k}}{2} \frac{\partial r_{i}}{\partial x_{k}}=0 \quad \text { in } \Omega, \quad k=1, n_{d}
$$

where $n_{d}$ is the number of space dimensions of the problems (i.e. $n_{d}=3$ for $3 \mathrm{D}$ ) and

$$
r_{i}:=-\rho \frac{\partial^{2} u_{i}}{\partial t^{2}}+\frac{\partial \sigma_{i j}}{\partial x_{j}}+b_{i}
$$

In Equation (23), $\rho$ is density, $t$ is time, $\sigma_{i j}$ are stresses, $b_{i}$ are body forces, and $h_{k}$ are characteristic length distances of an arbitrary prismatic domain where equilibrium of forces is considered. Equations (22) and (23) are completed with adequate boundary conditions and constitutive equations. 
Employing a standard split of stresses into deviatoric and volumetric (pressure) parts, $s_{i j}$ and $p$, respectively

$$
\sigma_{i j}=s_{i j}+p \delta_{i j}
$$

the governing FIC equations of the mixed displacement-pressure formulation can be obtained in the following form [13]:

$$
\begin{gathered}
-\rho \frac{\Delta v_{i}}{\Delta t}+\frac{\partial s_{i j}}{\partial x_{j}}+\frac{\partial p}{\partial x_{i}}+b_{i}-\frac{h_{k}}{2} \frac{\partial r_{i}}{\partial x_{k}}=0, \quad \frac{\Delta u_{i}}{\Delta t}-v_{i}^{n+1 / 2}=0 \\
\frac{\Delta p}{K}-\frac{\partial\left(\Delta u_{i}\right)}{\partial x_{i}}-\sum_{i=1}^{n_{d}} \tau_{i} \frac{\partial r_{i}}{\partial x_{i}}=0
\end{gathered}
$$

where $K$ is the bulk modulus, $\varepsilon_{v}$ is the volumetric strain and $\tau_{i}$ are intrinsic time parameters given by

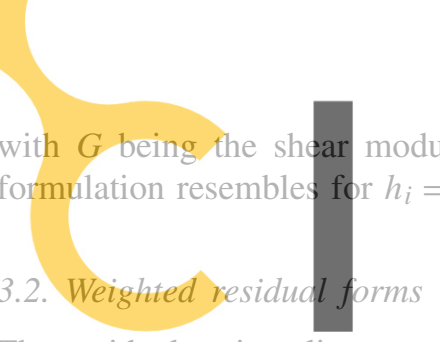

$$
\tau_{i}=\frac{3 h_{i}^{2}}{8 G}
$$
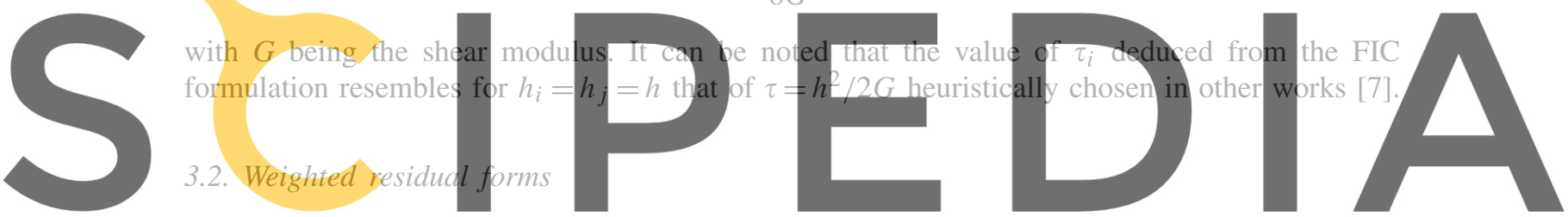

The residual $r_{i}$ is split now as

Register for free at https//www.scipedia.com to download the version without the watermark

$$
r_{i}=\pi_{i}+\frac{\partial \rho}{\partial x_{i}}
$$

where

$$
\pi_{i}=-\rho \frac{\partial v_{i}}{\partial t}+\frac{\partial s_{i j}}{\partial x_{i}}+b_{i}
$$

Note that $\pi_{i}$ is the part of $r_{i}$ not containing the pressure gradient and may be considered as the negative of a projection of the pressure gradient. In a discrete setting, the terms $\pi_{i}$ can be considered belonging to a space orthogonal to that of the pressure gradient terms. This is similar to the stabilization procedure based on a SGS method suggested in References [10-12].

Finally, the weighted residual form of the governing equations is written in the form

$$
\begin{array}{r}
\int_{\Omega} \delta u_{i} \rho \frac{\Delta v_{i}}{\Delta t} \mathrm{~d} \Omega+\int_{\Omega} \delta \varepsilon_{i j} \sigma_{i j} \mathrm{~d} \Omega-\int_{\Omega} \delta u_{i} b_{i} \mathrm{~d} \Omega-\int_{\Gamma_{t}} \delta u_{i} \bar{t}_{i} \mathrm{~d} \Gamma_{t}=0 \\
\int_{\Omega} \delta v_{i}\left[\frac{\Delta u_{i}}{\Delta t}-v_{i}\right] \mathrm{d} \Omega=0
\end{array}
$$




$$
\begin{array}{r}
\int_{\Omega} q\left(\frac{\Delta p}{K}-\frac{\partial\left(\Delta u_{i}\right)}{\partial x_{i}}\right) \mathrm{d} \Omega+\int_{\Omega}\left[\sum_{i=1}^{n_{d}} \frac{\partial q}{\partial x_{i}} \tau_{i}\left(\frac{\partial p}{\partial x_{i}}+\pi_{i}\right)\right] \mathrm{d} \Omega=0 \\
\int_{\Omega}\left[\sum_{i=1}^{n_{d}} w_{i} \tau_{i}\left(\frac{\partial p}{\partial x_{i}}+\pi_{i}\right)\right] \mathrm{d} \Omega=0
\end{array}
$$

The stabilization of the momentum equation is necessary in convection-dominated problems, this is not relevant for solid mechanics problems, so the additional terms involving the space derivatives of the characteristic lengths have been omitted in Equation (29a). On the contrary, the stabilization term given by the last integral is essential in Equation (29c).

\subsection{Finite element discretization}

Introduction of finite element discretization of the displacements, the pressure and the pressure gradient projection gives the following system of discretized equations:

$$
\mathbf{M} \frac{\Delta \overline{\mathbf{v}}}{\Delta t}-\mathbb{R}_{v}+\mathbf{f}_{d}=0
$$

\section{(30a)}
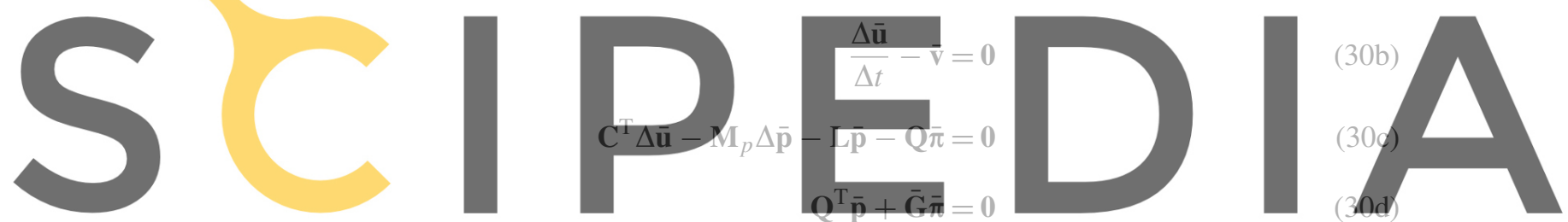

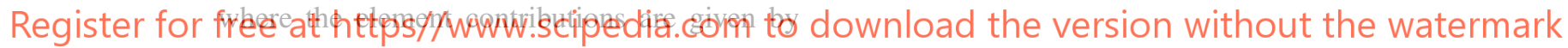

$$
\begin{aligned}
& \mathbb{L}=\int_{\Omega}(\nabla \mathbf{N})^{\mathrm{T}} \tau \nabla \mathrm{Nd} \Omega, \quad \overline{\mathrm{G}}=\int_{\Omega} \mathrm{N}^{\mathrm{T}} \tau \mathrm{N} \mathrm{d} \Omega \\
& \mathbf{Q}=\int_{\Omega}(\nabla \mathbf{N})^{\mathrm{T}} \tau \mathbf{N} \mathrm{d} \Omega, \quad \mathbf{f}_{d}=\int_{\Omega} \mathbf{B}^{\mathrm{T}} \boldsymbol{\sigma} \mathrm{d} \Omega
\end{aligned}
$$

where

$$
\tau=\left[\begin{array}{ccc}
\tau_{1} & 0 & 0 \\
0 & \tau_{2} & 0 \\
0 & 0 & \tau_{3}
\end{array}\right]
$$

The matrices $\mathbf{M}, \mathbf{M}_{p}, \mathbf{C}$ and $\mathbf{R}_{v}$ are defined in Equation (6). In the discretization procedure the same interpolation has been assumed for all the discretized fields: $\mathbf{N}_{v}=\mathbf{N}_{p}=\mathbf{N}_{\pi}=\mathbf{N}$.

The consistent definition of the characteristic length parameters is still an open question. In advective-diffusive and fluid flow problems it is usual to accept that the characteristic length vector has the direction of the velocity vector (this is the so-called streamline upwind Petrov-Galerkin or SUPG assumption [24]). In the examples presented in this paper, we have obtained good results using a simpler definition of the characteristic lengths with $h_{i}=h_{j}=h_{\min }$, where $h_{\min }$ is the smallest of the element (triangles or tetrahedra) heights. 


\subsection{Solution scheme}

A four-step semi-implicit time integration algorithm can be derived from Equations (30a)-(30d) as follows:

(i) Compute the nodal velocities $\overline{\mathbf{v}}^{n+1 / 2}$

$$
\overline{\mathbf{v}}^{n+1 / 2}=\overline{\mathbf{v}}^{n-1 / 2}+\Delta t \mathbf{M}^{-1}\left(\mathbf{R}_{v}^{n}-\mathbf{f}_{d}^{n}\right)
$$

(ii) Compute the nodal displacements $\overline{\mathbf{u}}^{n+1}$

$$
\overline{\mathbf{u}}^{n+1}=\overline{\mathbf{u}}^{n}+\Delta t \overline{\mathbf{v}}^{n+1 / 2}
$$

(iii) Compute the nodal pressures $\overline{\mathbf{p}}^{n+1}$

$$
\overline{\mathbf{p}}^{n+1}=\left[\mathbf{M}_{p}-\mathbf{L}\right]^{-1}\left[\Delta t \mathbf{C}^{\mathrm{T}} \overline{\mathbf{v}}^{n+1 / 2}+\mathbf{M}_{p} \overline{\mathbf{p}}^{n}-\mathbf{Q} \bar{\pi}^{n}\right]
$$

(iv) Compute the nodal projected pressure gradients $\bar{\pi}^{n+1}$

$$
\bar{\pi}^{n+1}=-\overline{\mathbf{G}}^{-1} \mathbf{Q}^{\mathrm{T}} \overline{\mathbf{p}}^{n+1}
$$

In above matrices, $\mathbf{M}, \mathbf{M}_{p}, \mathbf{L}, \mathbf{C}, \mathbf{Q}$ and $\overline{\mathbf{G}}$ are evaluated at $t^{n+1}$ and

$$
\mathbf{f}_{d}^{n}=\int_{\Omega}\left[\mathbf{B}^{\mathrm{T}} \boldsymbol{\sigma}\right]^{n} \mathrm{~d} \Omega
$$

where the stresses $\boldsymbol{\sigma}^{n}$ are obtained by consistent integration of the adequate (non-linear) constitutive law.

Note that steps (i), (ii) and (iv) are fully explicit. A fully explicit algorithm can be obtained by computing $\overline{\mathbf{p}}^{n+1}$ from step (iii) in Equation (33c) as follows:

$$
\overline{\mathbf{p}}^{n+1}=\mathbf{M}_{p}^{-1}\left[\Delta t \mathbf{C}^{\mathrm{T}} \overline{\mathbf{v}}^{n+1 / 2}+\left(\mathbf{M}_{p}-\mathbf{L}\right) \overline{\mathbf{p}}^{n}-\mathbf{Q} \pi^{n}\right]
$$

Obviously, the explicit solution is efficient if diagonal forms of matrices $\mathbf{M}_{p}, \mathbf{M}$ and $\overline{\mathbf{G}}$ are used. The explicit solution is possible if certain compressibility is assumed, i.e. $K \neq 0$. If $K \rightarrow \infty$ pressure must be obtained using the implicit scheme given by Equation (33c).

\section{NUMERICAL RESULTS}

\subsection{Impact of a cylindrical bar}

The problem analysed is the impact of a cylindrical bar with initial velocity of $227 \mathrm{~m} / \mathrm{s}$ into a rigid wall. The bar has an initial length $32.4 \mathrm{~mm}$ and initial radius $3.2 \mathrm{~mm}$. Material properties of the bar are typical of copper: density $\rho=8930 \mathrm{~kg} / \mathrm{m}^{3}$, Young's modulus $E=1.17 \times 10^{5} \mathrm{MPa}$, Poisson's ratio $v=0.35$, initial yield stress $\sigma_{\mathrm{Y}}=400 \mathrm{MPa}$ and isotropic hardening modulus $H=100 \mathrm{MPa}$. A time period of $80 \mu$ s has been analysed.

Figure 1 shows 2D and 3D solutions using triangular and tetrahedral elements based on equalorder interpolation for displacement and pressure-both solutions exhibit volumetric locking. Figure 2 shows correct numerical solution obtained using quadrilateral elements, a mixed displacement-pressure formulation with constant discontinuous pressure in each element. This 


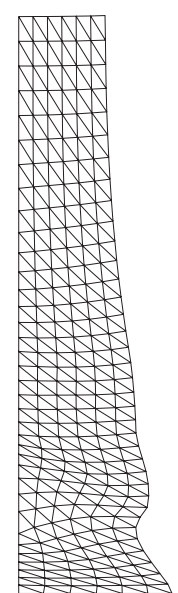

(a)

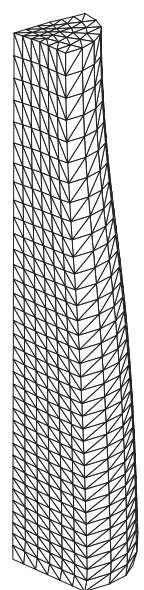

(b)

Figure 1. Final deformed mesh for standard displacement solution with locking: (a) 2D solution using axisymmetric triangular elements; and (b) 3D solutions using tetrahedra elements.

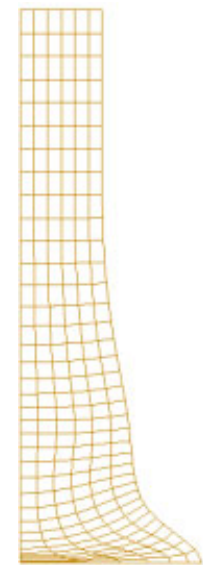

(a)

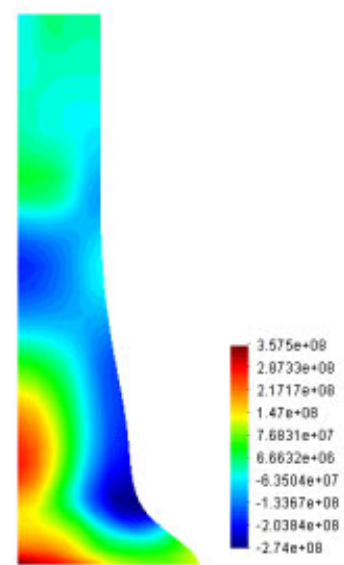

(b)

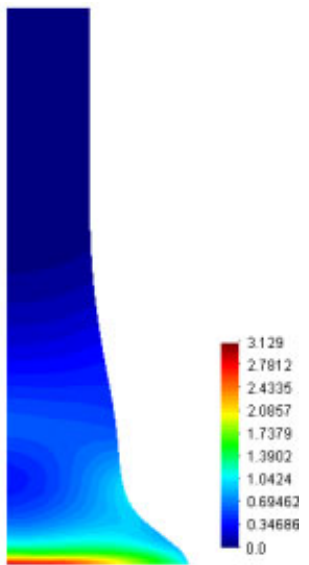

(c)

Figure 2. 2D explicit solution using mixed formulation with quadrilateral elements: (a) deformed mesh; (b) pressure distribution; and (c) effective plastic strain distribution.

solution is used as a reference of comparison for the CBS stabilized solution using triangular, tetrahedral, quadrilateral and hexahedral elements with equal-order displacement and pressure interpolation. The CBS results for triangles and tetrahedra are also compared to the results obtained using the alternative stabilized algorithm based on FIC. Results obtained using the CBS algorithm and quadrilateral elements with equal-order displacement and pressure interpolation are shown in Figure 3. Figures 4 and 5 show results obtained using triangular elements with the CBS algorithm. We can see that the solutions are locking-free, and the distribution and values 


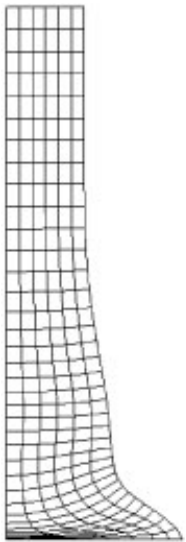

(a)

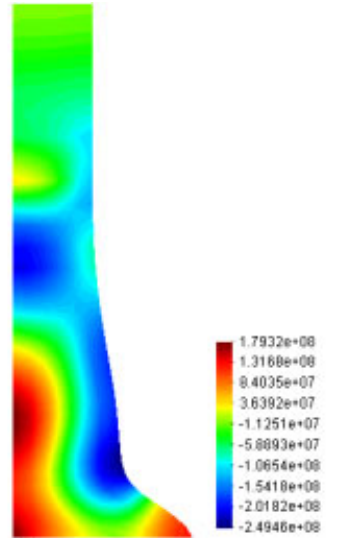

(b)

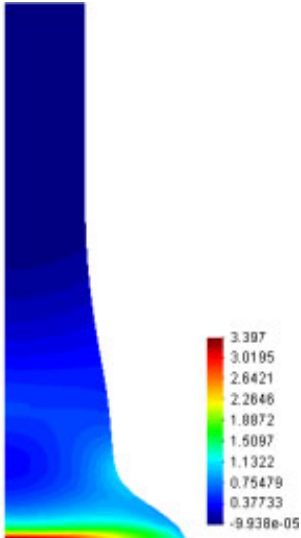

(c)

Figure 3. $2 \mathrm{D}$ explicit solution using the CBS algorithm $\left(\Delta t=0.60 \Delta t_{\mathrm{cr}}\right)$ with quadrilateral elements: (a) deformed mesh; (b) pressure distribution; and (c) effective plastic strain distribution.

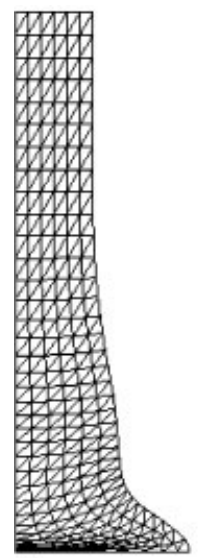

(a)

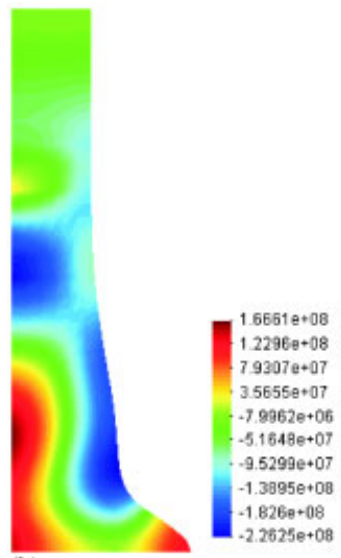

(b)

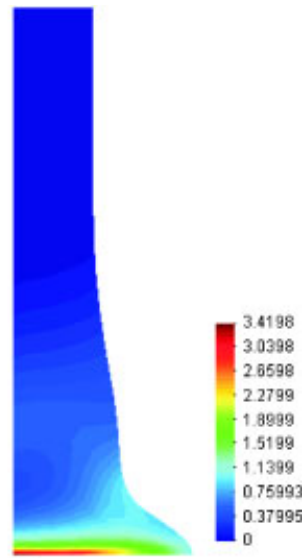

(c)

Figure 4. $2 \mathrm{D}$ solution using the CBS algorithm $\left(\Delta t=0.60 \Delta t_{\mathrm{cr}}\right)$ with triangular elements: (a) deformed mesh; (b) pressure distribution; and (c) effective plastic strain distribution.

of the pressure and effective plastic strain are consistent with those obtained using quadrilateral elements as shown in Figure 2. The two cases of analysis with the triangular mesh have different time steps, taken as 0.6 and 0.15 of the critical time step, respectively. Influence of the time step has been studied. Figure 6 shows the results obtained with triangular elements with the FIC stabilization, with $\tau$ taken as $\alpha h^{2} / G$ with $\alpha=0.01$. 3D solutions obtained using tetrahedra with the CBS and FIC stabilizations are shown in Figures 7 and 8, respectively.

Different 2D and 3D solutions have been compared in Figures 9 and 10. Figure 9 shows the pressure variation along the axis of symmetry and Figure 10 presents the pressure variation 


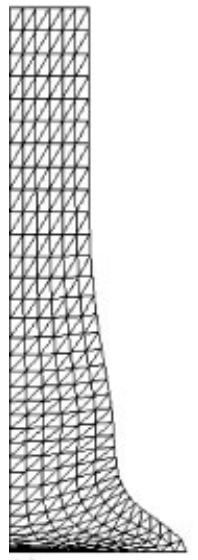

(a)

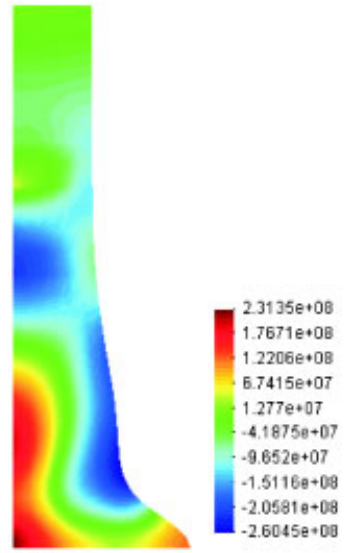

(b)

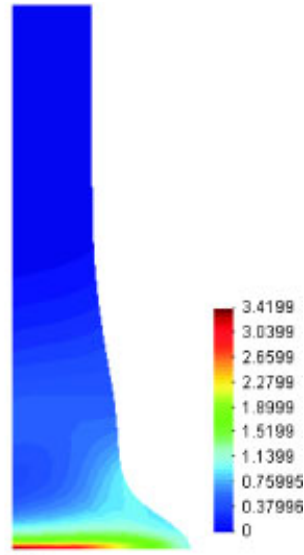

(c)

Figure 5. 2D solution using the CBS algorithm $\left(\Delta t=0.15 \Delta t_{\mathrm{cr}}\right)$ with triangular elements:

(a) deformed mesh; (b) pressure distribution; and (c) effective plastic strain distribution.

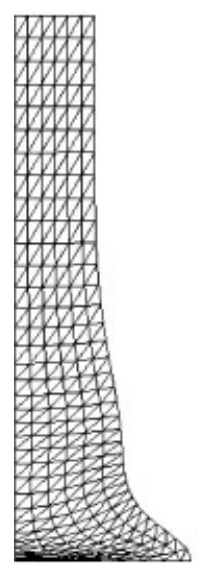

(a)

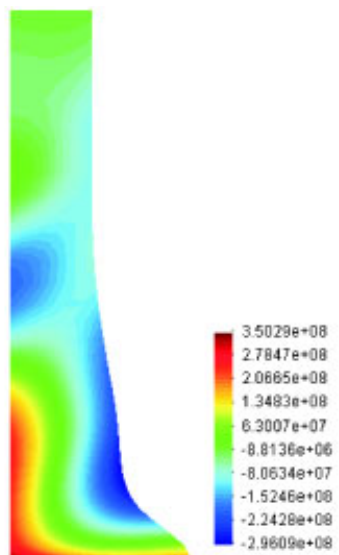

(b)

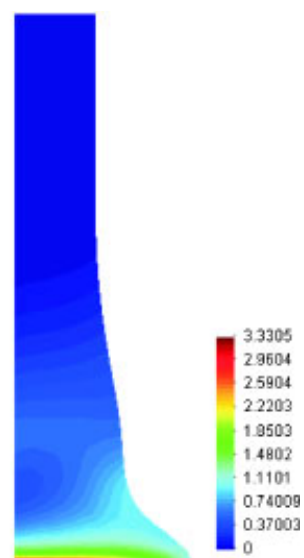

(c)

Figure 6. 2D solution using the FIC formulation $(\alpha=0.01)$ with triangular elements: (a) deformed mesh; (b) pressure distribution; and (c) effective plastic strain distribution.

along the generatrix. Although the pressures generally agree there is some divergence especially at the end of the axis of symmetry (Figure 9), which could indicate that a certain error is introduced by the approximate treatment of the traction boundary conditions in the simplified split algorithm.

\subsection{Sidepressing of a cylinder}

A cylinder $100 \mathrm{~mm}$ long with a radius of $100 \mathrm{~mm}$ is subjected to sidepressing between two rigid plane dies. It is compressed to $100 \mathrm{~mm}$. The material properties are the following: $E=217 \mathrm{GPa}$, 


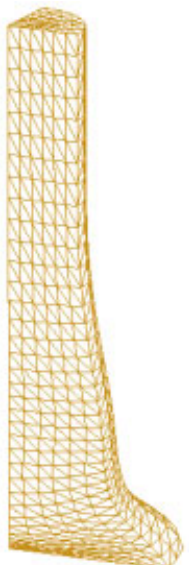

(a)

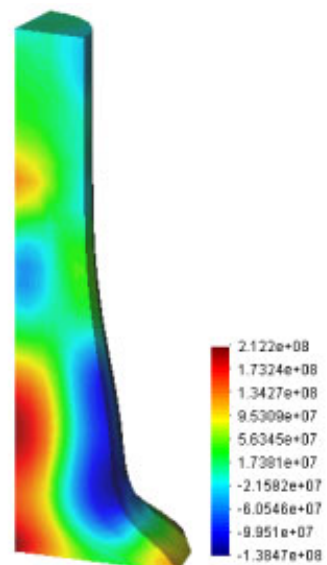

(b)

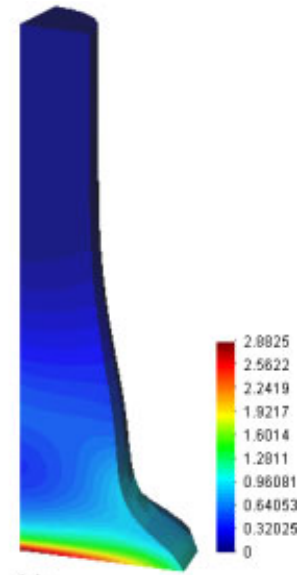

(c)

Figure 7. 3D using the CBS formulation with tetrahedral elements: (a) deformed mesh; (b) pressure distribution; and (c) effective plastic strain distribution.

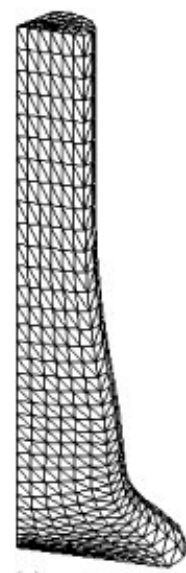

(a)

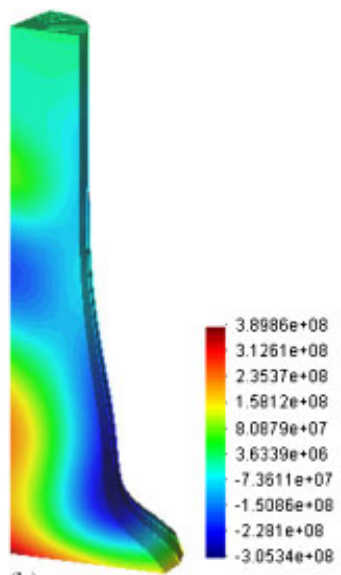

(b)

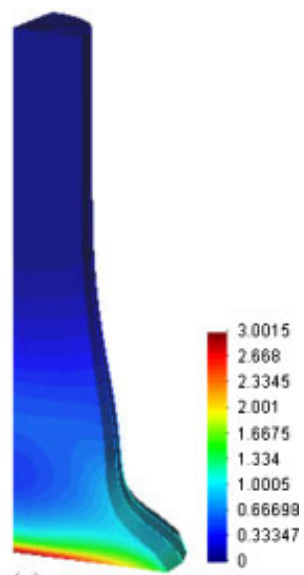

(c)

Figure 8. 3D solution using the FIC formulation $(\alpha=0.01)$ with tetrahedral elements: (a) deformed mesh; (b) pressure distribution; and (c) effective plastic strain distribution.

$v=0.3, \rho=7830 \mathrm{~kg} / \mathrm{m}^{3}, \sigma_{0}=170 \mathrm{MPa}, H=30 \mathrm{MPa}$, friction coefficient $=0.2$. The die velocity is assumed to be $2 \mathrm{~m} / \mathrm{s}$. Initial set-up is shown in Figure 11. A quarter of a cylinder was discretized with hexahedra or tetrahedra. Contact between the deformed material and die is treated using the penalty method.

Figure 12 shows the results obtained using hexahedrals mixed formulation with constant pressure and trilinear displacement interpolation. The results are shown in the form of deformed shape with distribution of the effective plastic strain and pressure. These results will be treated as the reference ones for other solutions. Results obtained using the CBS algorithm and hexahedral 


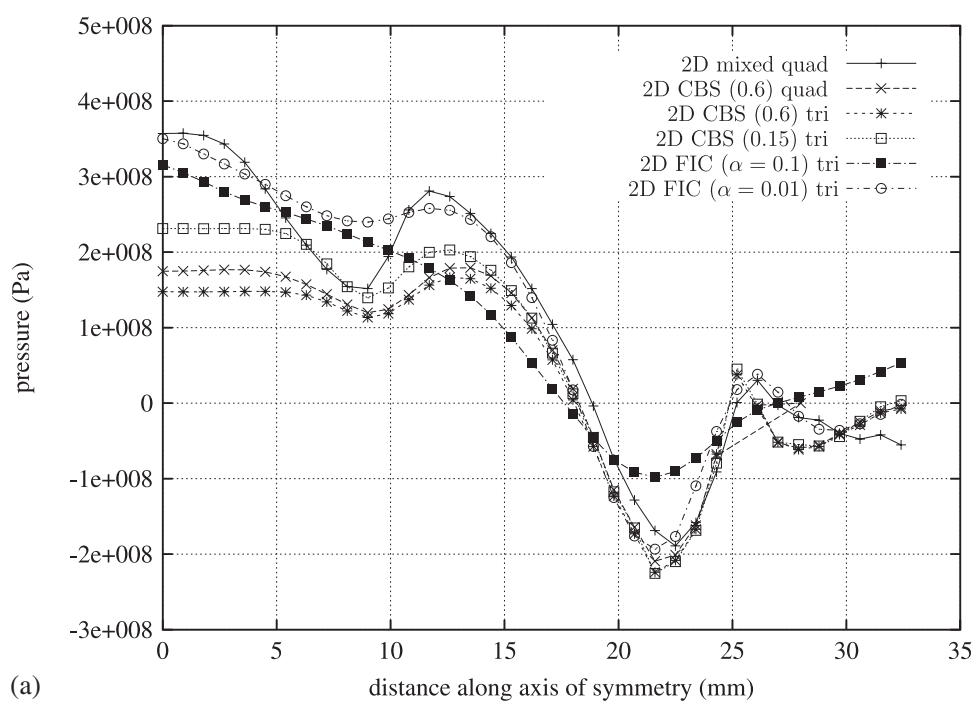

(a)

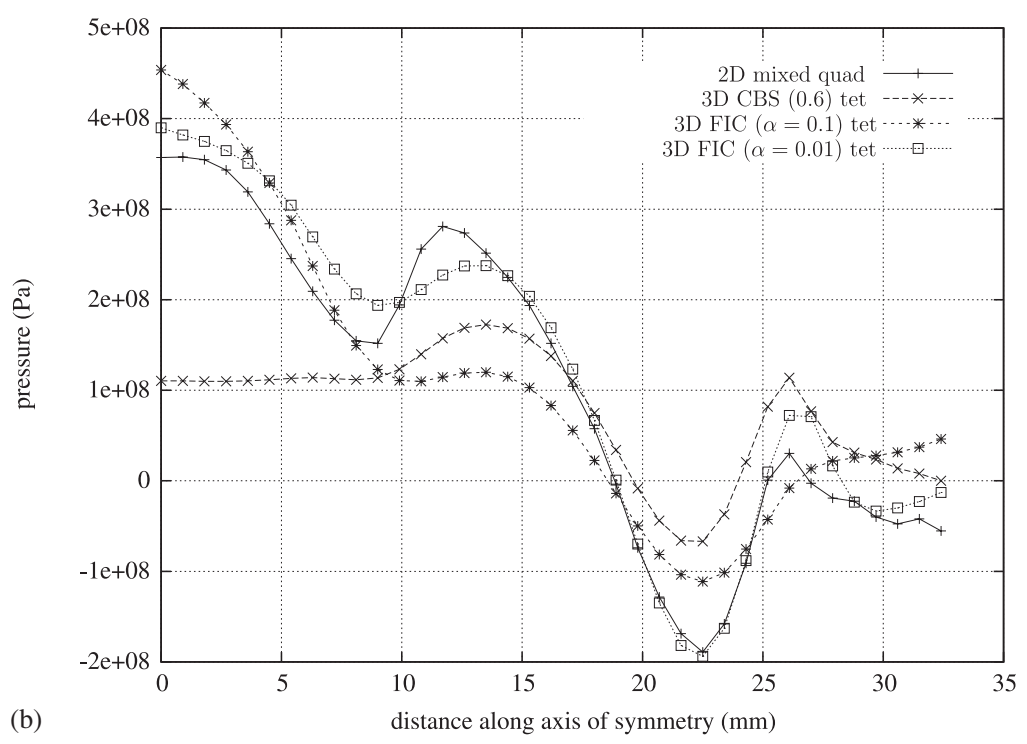

Figure 9. Pressure distribution along the axis of symmetry in: (a) 2D solutions; and (b) 3D solutions.

elements with equal-order displacement and pressure interpolation are shown in Figure 13. Figures 14 and 15 show the results obtained using the CBS algorithm for two different meshes of tetrahedra. The results obtained with the FIC stabilization are shown in Figure 16. Quite a good agreement can be seen between different solutions presented. Variation of pressure along the line ABCDEA defined in Figure 17 has been presented in Figure 18. Figure 18 confirms a good agreement between stabilized solutions with the reference solution. 

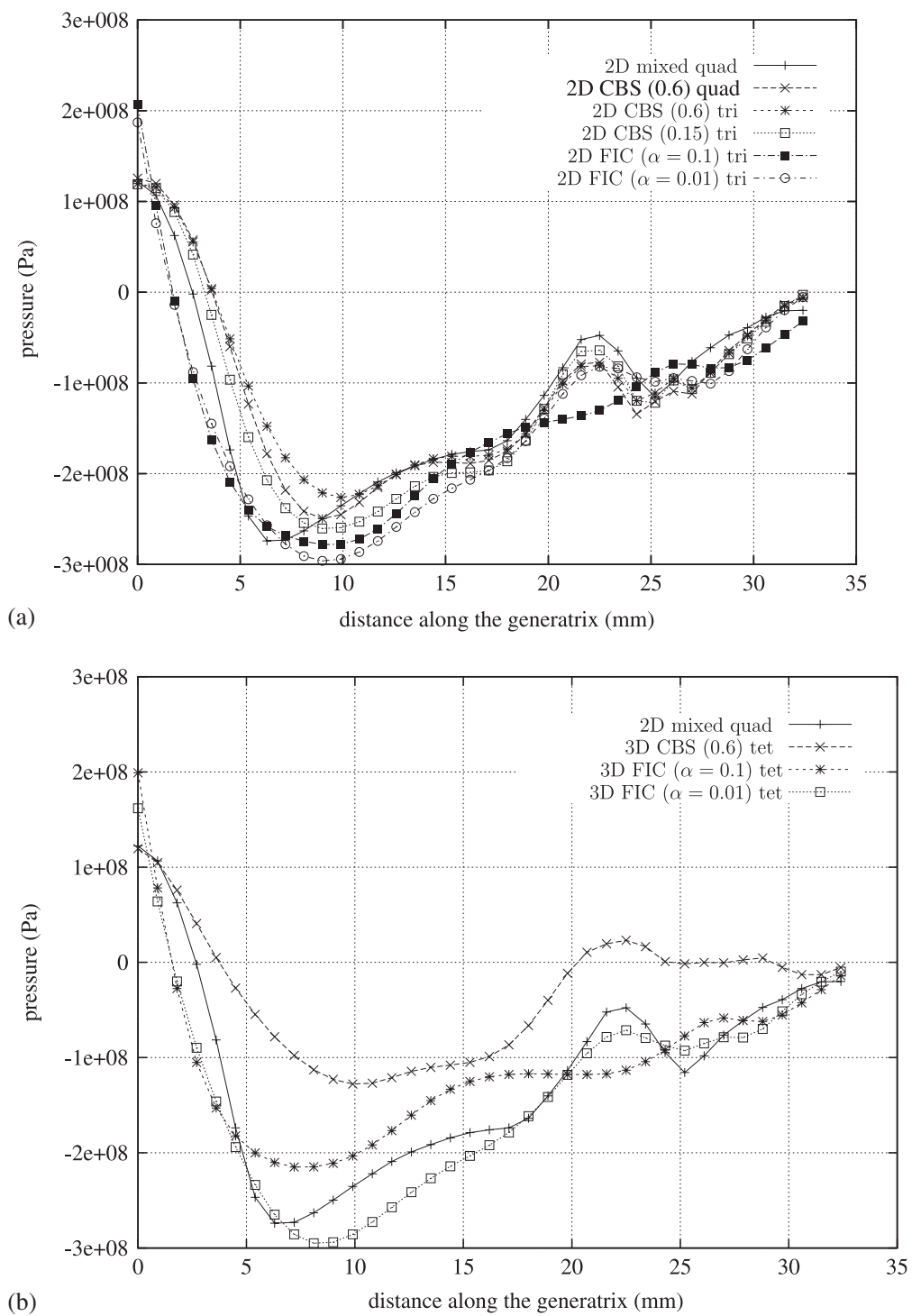

Figure 10. Pressure distribution along the generatrix in: (a) 2D solutions; and (b) 3D solutions.

\subsection{Backward extrusion}

Backward extrusion of a cylinder made of steel $16 \mathrm{MNCr} 5$ has been analysed. This is a benchmark example of the finite element program for forming simulation MARC/Autoforge [25]. The tooling and billet geometry are given in Figure 19(a). Initial material dimensions are the following: length $30 \mathrm{~mm}$ and diameter $30 \mathrm{~mm}$. Punch of diameter $20 \mathrm{~mm}$ has a prescribed stroke of $28 \mathrm{~mm}$. Material properties are as follows: Young's modulus $E=3.24 \times 10^{5} \mathrm{MPa}$, 


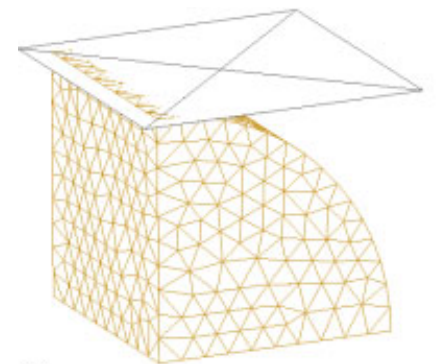

(a)

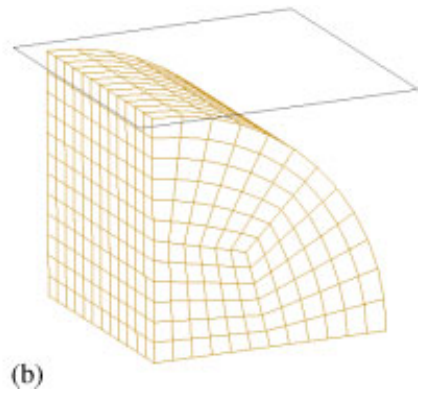

(b)

Figure 11. Sidepressing of a cylinder: (a) initial tetrahedral mesh; and (b) initial hexahedral mesh.

(a)

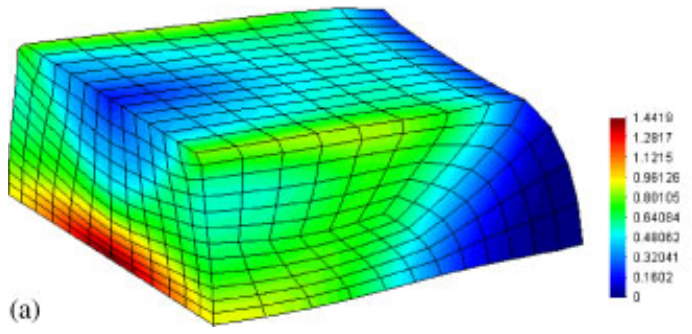

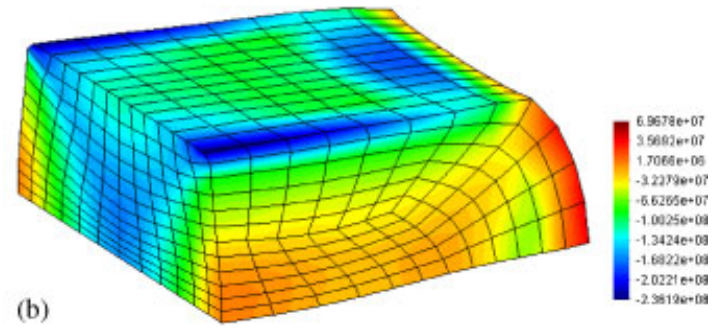

(b)

Figure 12. Sidepressing of a cylinder, mixed formulation, hexahedral mesh: (a) effective plastic strain; and (b) pressure distribution.
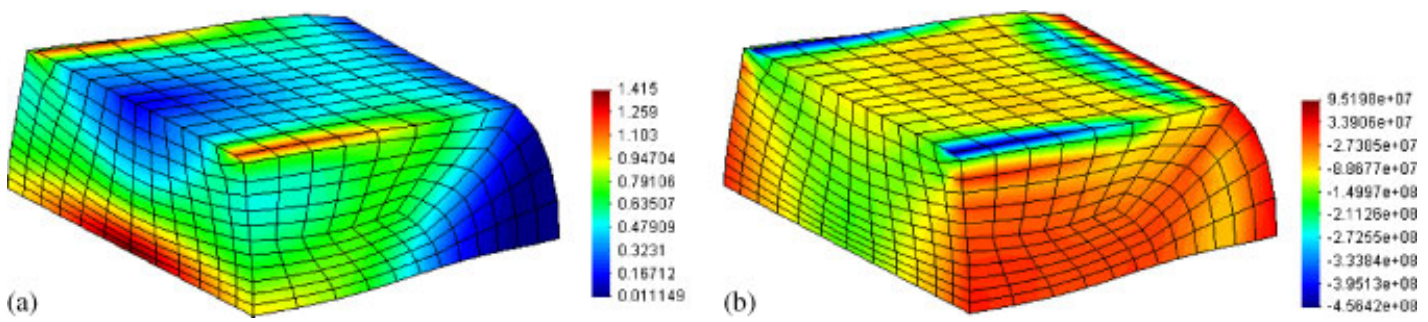

Figure 13. Sidepressing of a cylinder, CBS algorithm, hexahedral mesh: (a) effective plastic strain; and (b) pressure distribution.
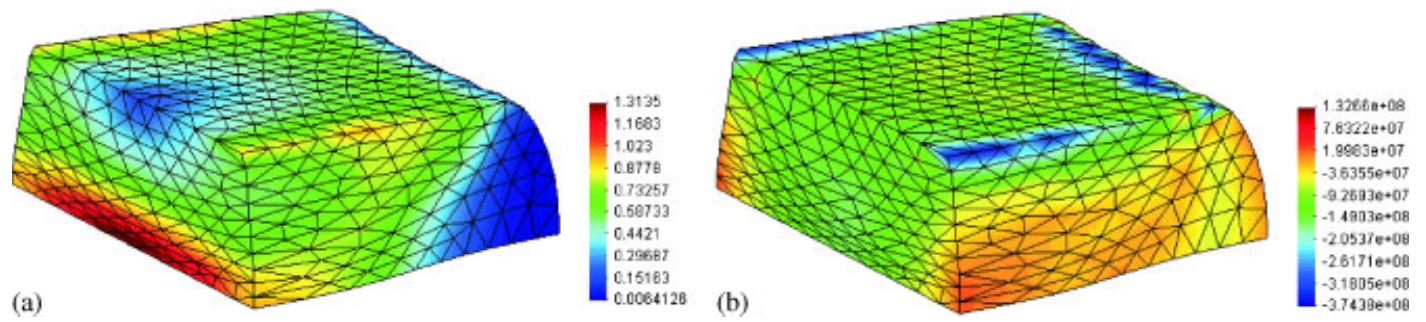

Figure 14. Sidepressing of a cylinder, CBS algorithm, tetrahedra, coarse mesh (4090 elements): (a) effective plastic strain; and (b) pressure distribution. 

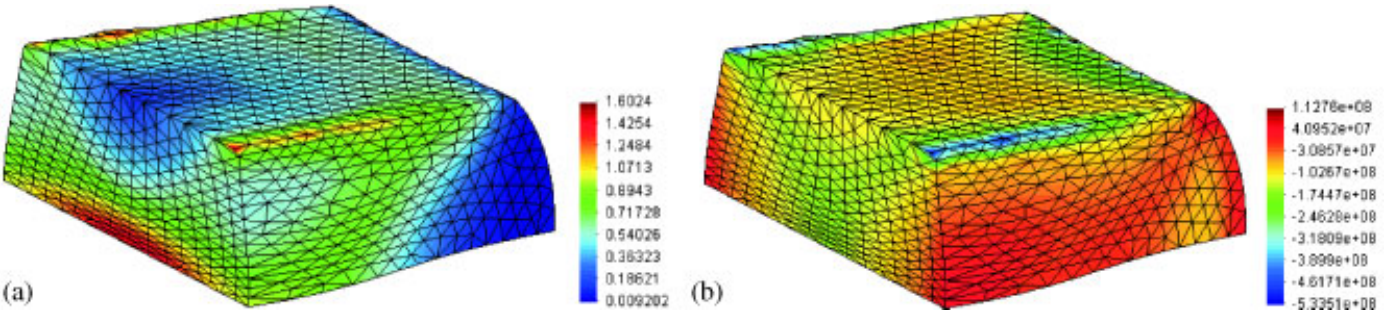

Figure 15. Sidepressing of a cylinder, CBS algorithm, tetrahedra, fine mesh (22 186 elements): (a) effective plastic strain; and (b) pressure distribution.

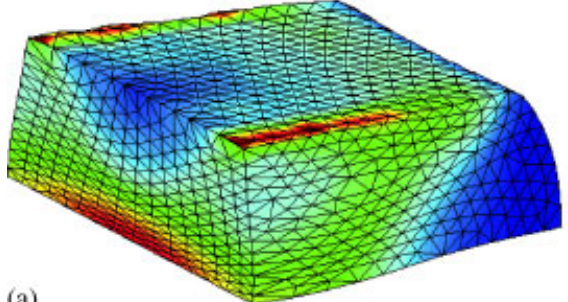

(a)

Figure 16. Sidepressing of a cylinder, FIC algorithm $(\alpha=0.1)$, tetrahedra, fine mesh (22 186 elements): (a) effective plastic strain; and (b) pressure distribution.

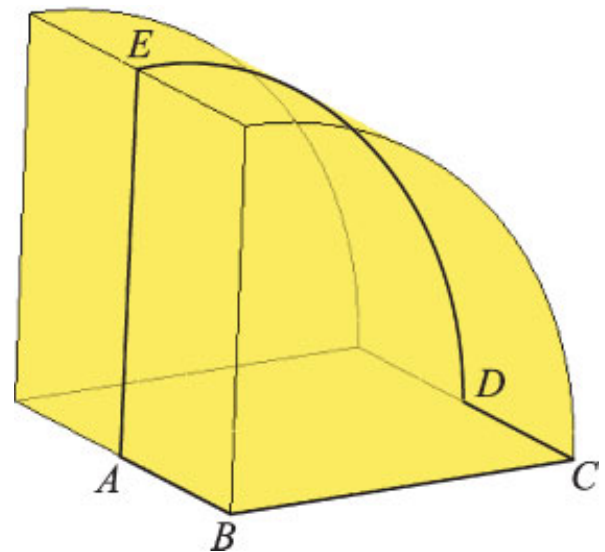

Figure 17. Definition of the line for comparison of pressure distribution.

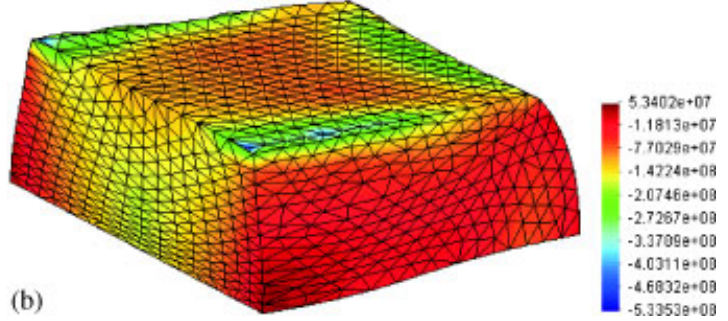

(b) 


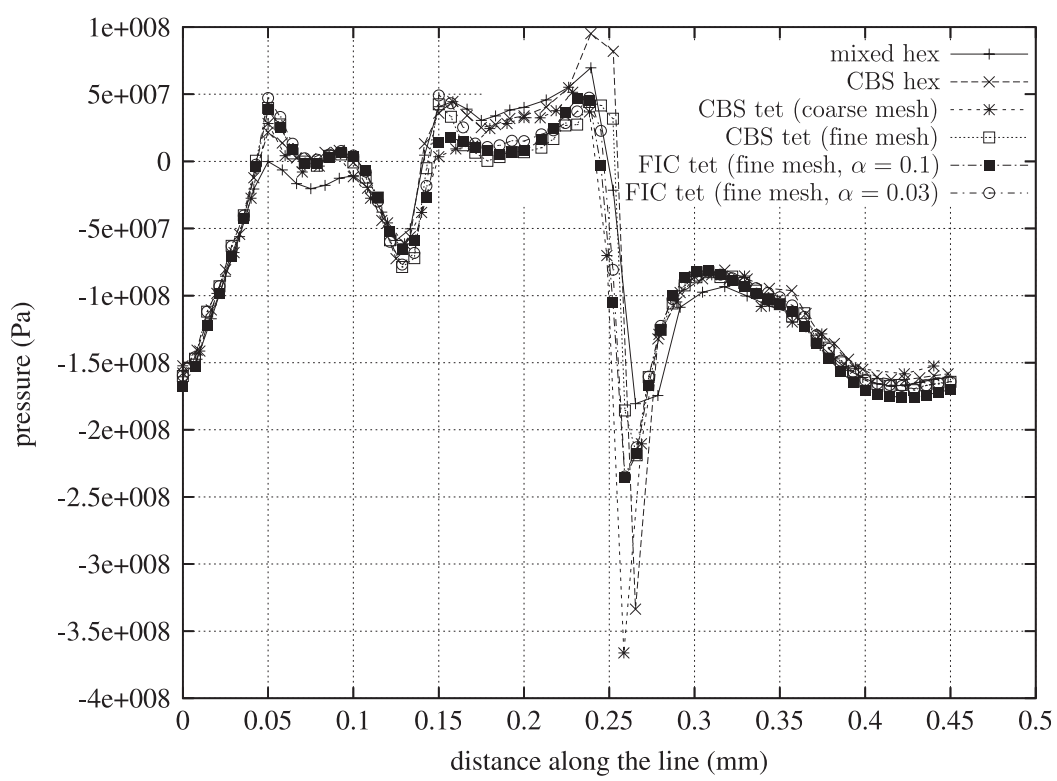

Figure 18. Pressure distribution along the line ABCDEA.

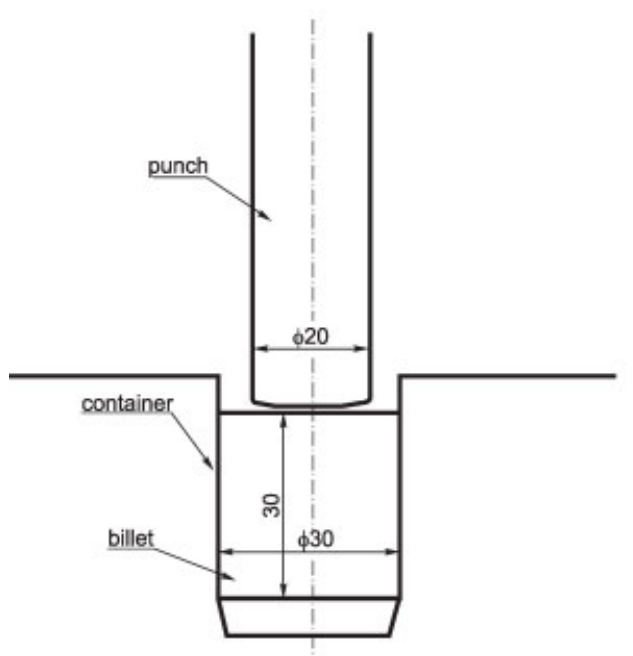

(a)

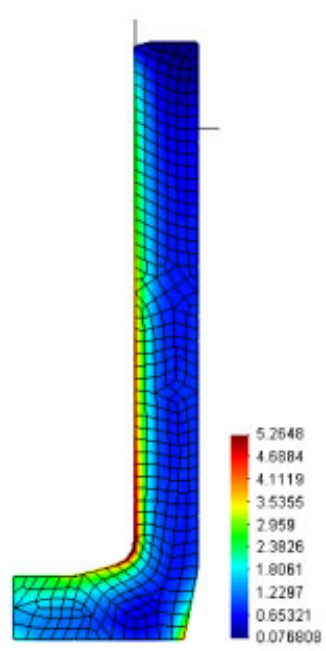

(b)

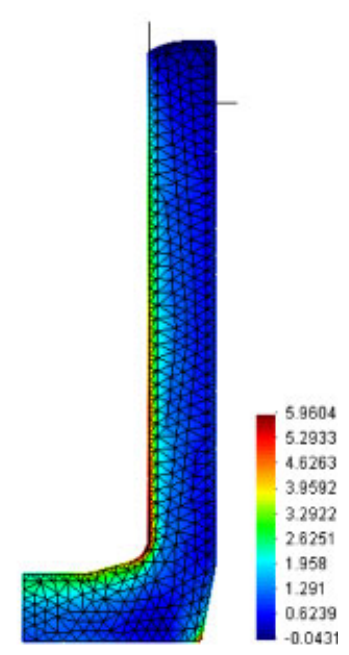

(c)

Figure 19. Backward extrusion: (a) geometry definition; (b) final deformed shape with effective plastic strain distribution, solution with quadrilaterals and mixed formulation; and (c) final deformed shape with effective plastic strain distribution, solution with triangles and the CBS algorithm. 
Poisson's coefficient $v=0.3$, material density $\rho=8120 \mathrm{~kg} / \mathrm{m}^{3}$, yield stress $\sigma_{\mathrm{Y} 0}=300 \mathrm{MPa}$ and hardening modulus $H=50 \mathrm{MPa}$. Friction between the material and tools is defined by the Coulomb friction coefficient $\mu=0.1$.

The simulation of the backward extrusion was carried out with remeshing employed to regenerate the meshes when element distortion was excessive. Figures 19(b) and (c) show the results in the form of the final deformed shape with the distribution of the effective plastic strain obtained using quadrilaterals and mixed formulation, and using triangles and the CBS algorithm, respectively. The results are in a good agreement with the solution given in Reference [25]. This example demonstrates use of the CBS algorithm in simulation of bulk forming processes.

\section{CONCLUSIONS}

The characteristic-based split algorithm provides the necessary stabilization for elements not satisfying BB conditions like equal-order displacement-pressure interpolation triangles, quadrilaterals, tetrahedra and hexahedra. The extension of the CBS algorithm to solid mechanics is straightforward. Its implementation in the explicit dynamic finite element program allows the simulation of solid mechanics problems with quasi-incompressible deformation of materials typical for bulk metal forming problems, for instance. The results obtained using the CBS algorithm are in quite a good agreement with the results obtained using other methods.

\section{ACKNOWLEDGEMENTS}

The authors are thankful to Professor O. C. Zienkiewicz for inspiration, continuous encouragement and many useful discussions.

\section{REFERENCES}

1. Zienkiewicz OC, Taylor RL. The Finite Element Method (5th edn). Butterworth-Heinemann: Oxford, 2000.

2. Babuška I. The finite element method with Lagrangian multipliers. Numerische Mathematik 1973; 20:179-192.

3. Brezzi F. On the existence, uniqueness and approximation of saddle-point problems arising from Lagrange multipliers. Revue française d'Automatique informatique recherche opérationalle Ser. Rouge Analyse numérique 1974; 8(R-2):129-151.

4. Zienkiewicz OC, Qu S, Taylor RL, Nakazawa S. The patch test for mixed formulations. International Journal for Numerical Methods in Engineering 1986; 23:1873-1883.

5. Zienkiewicz OC, Taylor RL. The finite element patch test revisited: a computer test for convergence, validation and error estimates. Computer Methods in Applied Mechanics and Engineering 1997; 149:523-544.

6. Brezzi F, Pitkäranta J. On the stabilization of finite element approximations of the Stokes problem. In Efficient Solution of Elliptic Problems, Hackbusch W (ed.), Notes on Numerical Fluid Mechanics. Vieweg: Wiesbaden, 1984.

7. Hughes TJR, Franca LP, Hulbert GM. A new finite element formulation for computational fluid dynamics: VIII. The Galerkin/least-squares method for advective-diffusive equations. Computer Methods in Applied Mechanics and Engineering 1989; 73:173-189.

8. Bonet J, Marriot H, Hassan O. Stability and comparison of different linear tetrahedral formulations for nearly incompressible explicit dynamic applications. International Journal for Numerical Methods in Engineering 2001; 50:119-133.

9. Bonet J, Marriot H, Hassan O. An average nodal deformation tetrahedron for large strain explicity dynamic applications. Communications in Numerical Methods in Engineering 2001; 17:551-561.

10. Codina R, Blasco J. Stabilized finite element method for transient Navier-Stokes equations based on pressure gradient projection. Computer Methods in Applied Mechanics and Engineering 2000; 182:287-300. 
11. Codina R. Stabilization of incompressibility and convection through orthogonal sub-scales in finite element methods. Computer Methods in Applied Mechanics and Engineering 2000; 190:1579-1599.

12. Chiumenti M, Valverde Q, Agelet de Saracibar C, Cervera M. A stabilized formulation for incompressible elasticity using linear displacement and pressure interpolations. Computer Methods in Applied Mechanics and Engineering 2002.

13. Oñate E, Taylor RL, Zienkiewicz OC, Rojek J. A residual correction method based on finite calculus. Engineering Computations 2003; 20:629-658.

14. Oñate E, Rojek J, Taylor RL, Zienkiewicz OC. Finite calculus formulation for incompressible solids using linear triangles and tetrahedra. International Journal for Numerical Methods in Engineering 2004; 59: 1473-1500.

15. Chorin AJ. A numerical method for solving incompressible viscous problems. Journal of Computational Physics 1967; 2:12-26.

16. Zienkiewicz OC, Codina R. A general algorithm for compressible and incompressible flow-part I: The split, characteristic-based scheme. International Journal for Numerical Methods in Fluids 1995; 20:869-885.

17. Zienkiewicz OC, Rojek J, Taylor RL, Pastor M. Triangles and tetrahedra in explicit dynamic codes for solids. International Journal for Numerical Methods in Engineering 1998; 43:565-583.

18. Rojek J, Zienkiewicz OC, Oñate E, Postek E. Advances in FE explicit formulation for simulation of metalforming processes. Journal of Materials Processing Technology 2001; 119(1-3):41-47.

19. Codina R. Pressure stability in fractional step finite element methods for incompressible flows. Journal of Computational Physics 2001; 170:112-140.

20. Rannacher R. On Chorin's Projection Method for the Incompressible Navier-Stokes Equations. In The Navier-Stokes Equations II-Theory and Numerical Methods. Proceedings of a Conference held Oberwolfach, Germany, 1991, Heywood JG, Masuda K, Rautmann R, Solonnikov SA (eds), Lecture Notes in Mathematics. Springer: Berlin, 1991; 167-183.

21. García Garino C, Oliver J. A numerical model for elastoplastic large strain problems. In Computational Plasticity, Owen DRJ et al (ed.). 1992.

22. Oñate E. Derivation of stabilized equations for advective-diffusive transport and fluid flow problems. Computer Methods in Applied Mechanics and Engineering 1998; 151:233-267.

23. Oñate E. A stabilized finite element method for incompressible flows using a finite increment calculus formulation. Computer Methods in Applied Mechanics and Engineering 2000; 182:355-370.

24. Brooks A, Hughes TJR. Streamline upwind/Petrov-Galerkin formulation for convection dominated flows with particular emphasis on the incompressible Navier-Stokes equations. Computer Methods in Applied Mechanics and Engineering 1982; 32:199-259.

25. MARC/Autoforge User's Guide 2.2. 1999. 